\title{
Phase Ib dose-finding trial of lapatinib plus pegylated liposomal doxorubicin in advanced HER2-positive breast cancer
}

\author{
Andrea Rocca ${ }^{1} \cdot$ Lorenzo Cecconetto $^{1} \cdot$ Alessandro Passardi $^{1} \cdot$ Elisabetta Melegari $^{1} \cdot$ Daniele Andreis $^{2} \cdot$ \\ Manuela Monti $^{2} \cdot$ Roberta Maltoni $^{1} \cdot$ Samanta Sarti $^{1} \cdot$ Elisabetta Pietri $^{1} \cdot$ Alessio Schirone $^{1} \cdot$ Francesco Fabbri $^{3}$. \\ Caterina Donati $^{4} \cdot$ Oriana Nanni $^{2} \cdot$ Anna Fedeli $^{1} \cdot$ Marina Faedi $^{1} \cdot$ Dino Amadori $^{1}$
}

Received: 13 October 2016 / Accepted: 6 March 2017 / Published online: 24 March 2017

(C) The Author(s) 2017. This article is published with open access at Springerlink.com

\begin{abstract}
Purpose Combination of anthracyclines with trastuzumab is hampered by cardiotoxicity. Pegylated liposomal doxorubicin and lapatinib could represent a safer alternative to combination therapy.

Methods In this phase Ib study with $3+3$ dose escalation design, patients with HER2-positive advanced breast cancer received pegylated liposomal doxorubicin $30 \mathrm{mg} / \mathrm{m}^{2}$ intravenously on day 1 plus lapatinib 1250 (level 1) or 1500 (level 2) mg/day orally on days 1-21 of each 21-day cycle. The aims were to establish the maximum tolerated dose at first cycle, and the activity and safety of multiple cycles.

Results Nine patients out of 11 enrolled were evaluable: 3 at level 1 and 6 at level 2. No dose-limiting toxicities occurred at dose level 1 , while 1 (grade 3 diarrhea) occurred at dose level 2, leading to the expansion of this cohort to 6 patients, with no further dose-limiting toxicities. Main grade 1-2 toxicities at first cycle were leucopenia, diarrhea, elevated transaminases, mucositis. Three patients had grade 3 toxicities at subsequent cycles,
\end{abstract}

Andrea Rocca

andrea.rocca@irst.emr.it

1 Department of Medical Oncology, Istituto Scientifico Romagnolo per lo Studio e la Cura dei Tumori (IRST) IRCCS, Meldola, Italy

2 Biostatistics and Clinical Trials Unit, Istituto Scientifico Romagnolo per lo Studio e la Cura dei Tumori (IRST) IRCCS, Meldola, Italy

3 Biosciences Laboratory, Istituto Scientifico Romagnolo per lo Studio e la Cura dei Tumori (IRST) IRCCS, Meldola, Italy

4 Oncology Pharmacy Laboratory, Istituto Scientifico Romagnolo per lo Studio e la Cura dei Tumori (IRST) IRCCS, Meldola, Italy including colitis, anorexia, stomatitis plus hand-foot syndrome. One partial response, 5 disease stabilizations, and 3 disease progressions were reported.

Conclusions Combination of pegylated liposomal doxorubicin and lapatinib is feasible and potentially active in pretreated HER2-positive advanced breast cancer patients.

Trial registration NCT02131506 (ClinicalTrials.gov identifier).

Keywords Breast cancer - HER2 - Targeted therapy · Lapatinib · Pegylated liposomal doxorubicin · Phase Ib

\section{Introduction}

Breast cancer (BC), the most frequent cancer worldwide among women, and one of the main leading causes of cancer death [1], consists of different subtypes according to biology, prognosis, and treatment response [2].

Before the advent of anti-human epidermal growth factor receptor 2 (HER2) therapies, survival of metastatic HER2-positive BC patients was quite poor [3]. The advent of targeted therapies has led to impressive improvements in survival [4]. Nonetheless, long-term outcome data show that only about $12 \%$ of patients maintains disease remission after 5 years of first-line trastuzumab-based therapy [5], and almost all patients eventually undergo disease progression and require additional therapies. Although evidence of benefit from HER2-targeted therapies beyond second-line treatment of metastatic BC is not definitive [6], its use is common in clinical practice and is recommended by treatment guidelines [7].

Anthracyclines are among the most active drugs in BC [8], and have been hypothesized [9] to be particularly effective in HER2-positive tumors [10]. Given the cardiac 
toxicity caused by both drugs $[11,12]$, the administration of conventional anthracyclines concomitantly with trastuzumab results in unacceptable rates of heart failure, especially in the metastatic setting [4], when long-term therapies are administered. As a consequence, antracyclines remain underused for HER2-positive metastatic BC in clinical practice, with only about $25 \%$ of eligible patients treated [13].

Liposomal formulations of doxorubicin appear to maintain similar efficacy with a reduced risk of cardiotoxicity compared to conventional doxorubicin [14]. Pegylated liposomal doxorubicin (PLD) has been approved by the European Medicines Agency for use as monotherapy in patients with metastatic BC in event of increased cardiac risk (PLD summary of product characteristics).

Lapatinib (L), a small molecule, reversible dual tyrosine kinase inhibitor selectively targeting HER2 and epidermal growth factor receptor (EGFR), is indicated for the treatment of adult patients with HER2-positive advanced or metastatic BC in combination with either capecitabine (following progression after therapy including anthracyclines, taxanes, and trastuzumab in the metastatic setting) or trastuzumab (in hormone receptor-negative disease progressing on prior trastuzumab) or with an aromatase inhibitor (in postmenopausal women with hormone receptor-positive disease, not currently intended for chemotherapy) (L summary of product characteristics). L causes low cardiotoxicity, usually consisting in asymptomatic, reversible decreases in left ventricular ejection fraction (LVEF), independently from pretreatment with anthracyclines or trastuzumab [15].

Preclinical work shows an additive cytotoxic effect of the combination of L and PLD in HER2-positive BC cell lines, and a potential synergy in other EGFR-positive/ HER2-negative cell lines [16].

We designed a phase Ib clinical trial with the aim to define the maximum tolerated dose (MTD) and the recommended phase-2 dose (RP2D) of this drug combination.

\section{Materials and methods}

\section{Study design}

This was an open label, phase Ib study, with standard $3+3$ dose escalation design.

The primary objectives were to define MTD, based on the dose-limiting toxicities (DLTs) observed during the first cycle of treatment, and RP2D.

Secondary objectives were to explore the antitumor activity, in terms of objective response rate and clinical benefit, and to assess the safety profile of multiple cycles of therapy, especially cardiac.
Four dose levels were planned: the starting dose level 1, with PLD $30 \mathrm{mg} / \mathrm{m}^{2}$ and L $1250 \mathrm{mg} /$ day; dose level 2, with PLD $30 \mathrm{mg} / \mathrm{m}^{2}$ and $\mathrm{L} 1500 \mathrm{mg} /$ day; dose level 3, with PLD $40 \mathrm{mg} / \mathrm{m}^{2}$ and L $1500 \mathrm{mg} /$ day; and dose level1, with PLD $30 \mathrm{mg} / \mathrm{m}^{2}$ and L $1000 \mathrm{mg} /$ day.

Cohorts of 3-6 patients were required for treatment at successive dose levels, starting at level 1 . If none of the initial 3 patients for each dose level developed a first-course DLT, the following cohort could start 1 dose level higher. If 1 of the initial 3 patients developed a first-course DLT, a maximum of 3 additional patients were to enter at the same level. MTD was defined as the dose level at which $\geq 33.3 \%$ of the patients experienced first-course DLT. If the cohort below MTD, i.e., RP2D, included 3 patients, the cohort was expanded to 6 patients.

DLT was defined as any of the following first-cycle events: grade (G) 4 neutropenia (absolute neutrophil count $[\mathrm{ANC}]<500$ cells $/ \mathrm{mm}^{3}$ ) for $\geq 5$ days, or febrile neutropenia (fever $\geq 38.5^{\circ} \mathrm{C}$ with $\mathrm{ANC}<1000$ cells/ $\mathrm{mm}^{3}$ ); G4 thrombocytopenia (platelet count $<25,000$ cells $/ \mathrm{mm}^{3}$ ), or $\mathrm{G} 3$ thrombocytopenia associated with a bleeding episode requiring platelet transfusion; $G \geq 3$ non-hematological toxicity; failure to readminister treatment within 14 days of the planned drug administration due to delayed recovery of treatment-related toxicity to $G \leq 1$ or baseline.

Toxicity was graded according to the Common Terminology Criteria for Adverse Events (CTCAE) version 3.0.

Treatment was discontinued in case of disease progression, unacceptable toxicity, appearance of intercurrent illnesses or clinical conditions which jeopardized continuation of therapy, or patient decision to withdraw from the study. If interruption of PLD was clinically indicated, patients could continue to receive L monotherapy.

\section{Study treatment}

Each 21-day cycle included L administered orally once daily ( $1 \mathrm{~h}$ before or after breakfast) on days $1-21$, and PLD administered intravenously (i.v.) in a 60 minutes' infusion on day 1 (the first dose was started more slowly to minimize the risk of infusion reactions). Thirty minutes before PLD administration, patients received dexamethasone $8 \mathrm{mg}$ i.v. and ondansetron $8 \mathrm{mg}$ i.v. Antiemetic medications were prescribed for the following days as needed. Patients were required to comply with the prescribing information for $\mathrm{L}$, including avoidance of inducers and strong inhibitors of CYP3A4; medications for increasing gastric $\mathrm{pH}$ may have been taken within $1 \mathrm{~h}$ of administration of L, if strictly needed. 


\section{Dose adjustments}

Treatment was temporarily withheld in the event of G4 hematological or $G \geq 3$ non-hematological toxicity or $G \geq 2$ left ventricular systolic dysfunction (LVSD). Delay of subsequent cycles occurred in case of $G \geq 2$ hematological on non-hematological toxicity on day 1 . Dose reductions were adjusted according to the worst toxicity occurred in the previous cycle, with a reduction of $1 \mathrm{~L}$ dose level and $25 \%$ of PLD dose in the event of febrile neutropenia, G4 thrombocytopenia or bleeding associated with thrombocytopenia, or $G \geq 3$ non-hematological toxicity. Definitive treatment interruptions were planned in case of G3 or G4 LVSD, G4 rash manifested as toxic epidermal necrolysis/ Stevens-Johnson's Syndrome, liver toxicity fulfilling Hy's law $($ ALT $>3 \times$ ULN and total bilirubin $>2.0 \times U L N)$.

\section{Safety and tumor assessments}

Baseline evaluation included medical history (with concomitant medications) and physical examination, vital parameters and ECOG performance status (PS), cardiologic examination including ECG and echocardiography, laboratory assessments (hematology and blood chemistry, including high-sensitivity troponin $\mathrm{T}$ [TnT-hs] and N-terminal pro b-type Natriuretic Peptide [NT-proBNP]), and tumor assessment with CT scan of the thorax and abdomen. Brain CT scan and bone scintigraphy, as well as other imaging studies, were performed if clinically indicated.

Complete blood count and blood chemistry were repeated weekly. Toxicity, physical examination, vital parameters, and PS were assessed before each cycle. Cardiac monitoring included biomarker measurement before (TnT-hs + NT-proBNP) and $24 \mathrm{~h}$ after (TnT-hs) PLD administration, as a correlative research evaluation, and ECG + cardiologic exam and echocardiogram (with measurement of LVEF) every 2 cycles of therapy.

Imaging studies for tumor evaluation were performed every third cycle and whenever clinically indicated.

\section{Patient population}

The main inclusion criteria were: histological or cytological diagnosis of BC; locally advanced (stage IIIB-IIIC) inoperable or metastatic (stage IV) disease; HER2 overexpression, defined as +3 staining in immunohistochemistry, or HER2 amplification at fluorescence in situ hybridization (FISH); age $\geq 18$ years; ECOG performance status $\leq 2$; life expectancy $\geq 12$ weeks; disease progression following prior therapy with taxane- and trastuzumab-containing regimens (if not contraindicated); previous cumulative doxorubicin dose $\leq 240 \mathrm{mg} / \mathrm{m}^{2}$; LVEF within the institutional range of normal; adequate bone marrow, liver, and renal functions; and ability to understand and willingness to sign a written informed consent document.

Exclusion criteria included: prior exposure to PLD or L, history of allergic reactions to compounds of similar chemical composition, prior treatment with anthracyclines within 1 year of study entry or prior disease progression while on anthracycline therapy, a pregnant or lactating status, non-compliance with adequate contraceptive measures for patients with reproductive potential, prior or concurrent history of other neoplasms, symptomatic brain metastases, and uncontrolled intercurrent illnesses.

All patients signed a written informed consent. The study was approved by the Scientific and the Ethics Committees of our Institute, and performed in accordance with the Declaration of Helsinki and the Good Clinical Practice guidelines. ClinicalTrials.gov identifier: NCT02131506.

\section{Statistical analysis}

Descriptive statistics are reported for patients' characteristics and response analysis. The Kaplan-Meier method was used to analyze the time-to-event endpoints. After visually checking homoscedasticity and normality of residual plots, a linear mixed effects model, with random intercepts and slopes, was fitted to evaluate changes of LVEF over time, with time as fixed effect and subjects as random effect. Analyses were done using R (R Core Team 2015) and the R lme4 package.

\section{Results}

Between February 2010 and June 2015, 11 patients were enrolled into the study, 9 of whom were evaluable. A 45-year-old woman with metastatic HER2-positive lobular carcinoma, pretreated with trastuzumab plus pertuzumab plus docetaxel for 2 cycles (in the Cleopatra trial), switched to vinorelbine plus trastuzumab because of an allergic reaction to docetaxel, was considered non-evaluable because of a grade 3 allergic reaction occurring immediately after the beginning of the first PLD infusion. A 65-year-old woman with metastatic HER2-positive lobular carcinoma, pretreated with 2 lines of trastuzumab-based therapy, withdrew her consent before starting therapy.

Characteristics of the 9 evaluable patients are reported in Table 1. Median age was 65 years (range 43-77). All patients had HER2-positive tumors of ductal histology, 7 of whom were hormone receptor positive, and 5 had Ki67 $\geq 20 \%$. Only 3 patients were pretreated with adjuvant chemotherapy, including anthracyclines in 2 cases, and 2 had adjuvant trastuzumab. All but 1 were pretreated for metastatic disease, including $\geq 2$ lines of trastuzumab-based treatment in most cases. The only patient not pretreated for 
Table 1 Patient and tumor characteristics (for the 9 evaluable patients)

\begin{tabular}{|c|c|}
\hline Variable & $\begin{array}{l}\text { Median } \\
\text { (range), or } \\
n(\%)\end{array}$ \\
\hline Age & $65(43-77)$ \\
\hline \multicolumn{2}{|l|}{ Performance status (ECOG) } \\
\hline 0 & $5(56)$ \\
\hline 1 & $4(44)$ \\
\hline Ductal histology & $9(100)$ \\
\hline \multicolumn{2}{|l|}{ Hormone receptors } \\
\hline \multicolumn{2}{|l|}{ Estrogen } \\
\hline Positive & $7(78)$ \\
\hline Negative & $2(22)$ \\
\hline \multicolumn{2}{|l|}{ Progesterone } \\
\hline Positive & $5(56)$ \\
\hline Negative & $4(44)$ \\
\hline \multicolumn{2}{|l|}{ Ki-67 } \\
\hline$<20 \%$ & $4(44)$ \\
\hline$\geq 20 \%$ & $5(55)$ \\
\hline HER2 status positive & $9(100)$ \\
\hline \multicolumn{2}{|l|}{ Previous (neo)adjuvant chemotherapy } \\
\hline With antracyclines & $2(22)$ \\
\hline Without antracyclines & $1(11)$ \\
\hline Previous (neo)adjuvant trastuzumab & $2(22)$ \\
\hline \multicolumn{2}{|c|}{ Previous lines of therapy for metastatic breast cancer } \\
\hline 0 & $1(11)$ \\
\hline 1 & $2(22)$ \\
\hline 2 & $3(33)$ \\
\hline 3 & $3(33)$ \\
\hline Trastuzumab plus taxane $^{\mathrm{a}}$ & $7(78)$ \\
\hline Trastuzumab plus endocrine agent & $2(22)$ \\
\hline Trastuzumab plus vinorelbine/capecitabine & $4(44)$ \\
\hline T-DM1 & $2(22)$ \\
\hline Neratinib plus capecitabine & $1(11)$ \\
\hline Cyclophosphamide plus docetaxel & $1(11)$ \\
\hline \multicolumn{2}{|l|}{ Number of metastatic sites } \\
\hline 1 & $4(44)$ \\
\hline$\geq 2$ & $5(55)$ \\
\hline \multicolumn{2}{|l|}{ Sites of metastases } \\
\hline Soft tissues & $2(22)$ \\
\hline Bone & $6(67)$ \\
\hline Viscera & $6(67)$ \\
\hline
\end{tabular}

${ }^{a}$ In two patients, an endocrine agent was substituted for paclitaxel as maintenance therapy, in combination with trastuzumab, after about 6 months of treatment and an objective response achievement

metastatic disease was a 75-year-old lady who had disease relapse right at the end of adjuvant trastuzumab (prior to T-DM1 registration); she received doxorubicin + cyclophosphamide for 4 cycles and 12 weekly administrations of paclitaxel in the adjuvant setting; at the time of relapse, she refused treatment-induced alopecia, and was not candidate to lapatinib + capecitabine according to the Italian lapatinib indication; therefore, after careful discussion, treatment within the trial appeared a good option and was acceptable for the patient. All had good performance status, although most had $\geq 2$ metastatic sites and visceral involvement.

Of the nine patients, three were enrolled at dose level 1. As none of these patients developed DLT at first cycle, the second cohort started at dose level 2. Among the first three patients of the latter cohort, 1 presented a DLT (G3 diarrhea at first cycle), leading to the expansion of the cohort to 6 patients, with no further occurrence of DLT.

The median number of treatment cycles were 7 for PLD (range 3-13) and 6 for L (range 1-26). The median dose intensity of PLD was $9.4 \mathrm{mg} / \mathrm{m}^{2} /$ week (range $8.3-10.0$ ). During the whole course of therapy, the median dose intensity of L was $1195 \mathrm{mg} /$ day (range 996-1500) (dose level 1: median 1195, range 996-1225; level 2: median 1220, range 1048-1500).

First cycle toxicities are reported in Table 2. One case of neutropenia and 1 of elevated transaminases represented the only G2 events at first dose level, with G1 leucopenia, nausea, anorexia, and diarrhea as additional side effects. Diarrhea was the predominant adverse event at dose level 2, with 1 case of G3 (DLT) and 2 cases of G1 and G2 each. The only other G2 event was a case of hyperbilirubinemia, which was not associated with elevation of transaminases, and therefore not considered a concern for serious liver injury. Other G1 events included leucopenia, neutropenia, anemia, fatigue, mucositis, epigastric pain.

Toxicities recorded along the whole treatment period are reported in Table 3. There were no G4 adverse events, and there was only $1 \mathrm{G} 3$ event represented by colitis at dose level 1, and $4 \mathrm{G} 3$ events at dose level 2, represented by diarrhea (already reported above as DLT at first cycle), hand-foot syndrome, mucositis, and anorexia, respectively. The most common G1-2 non-hematological adverse events were hand-foot syndrome, rash, fatigue, mucositis, epigastric pain, hyperbilirubinemia, transaminases elevation, and diarrhea. Hematological adverse events included G1-2 neutropenia in 6 patients, leucopenia in 5 patients, and anemia in 2 patients.

Regular comprehensive cardiologic evaluations were performed as described above. No cases of LVSD were reported, defined as a LVEF reduction to $\leq 50$ percentage points or a LVEF reduction of $>20$ percentage points of the baseline value (Fig. 1a), and actually no cases of LVEF reduction $\geq 10 \%$ points of the baseline value occurred. Median baseline LVEF was 67\% (range 59-77\%, mean $68 \%$ ) and median LVEF at nadir was 67\% (range 59-73\%, mean $66 \%$ ). The analysis of LVEF measures did not show significant changes over time $(p=0.26$, mixed effects model). Two patients prudently stopped PLD after 13 and 
Table 2 First cycle toxicity $(N=9$ patients $)$

\begin{tabular}{|c|c|c|c|c|c|c|c|c|c|c|c|c|}
\hline & \multicolumn{4}{|c|}{ Dose level 1} & \multicolumn{4}{|c|}{ Dose level 2} & \multicolumn{4}{|c|}{ Overall } \\
\hline & \multicolumn{4}{|l|}{$n^{\mathrm{a}}$} & \multicolumn{4}{|l|}{$n^{\mathrm{a}}$} & \multicolumn{4}{|l|}{$n^{\mathrm{a}}$} \\
\hline & G1 & $\mathrm{G} 2$ & G3 & G4 & G1 & $\mathrm{G} 2$ & G3 & G4 & G1 & $\mathrm{G} 2$ & G3 & G4 \\
\hline Leucopenia & 1 & 0 & 0 & 0 & 3 & 0 & 0 & 0 & 4 & 0 & 0 & 0 \\
\hline Neutropenia & 1 & 1 & 0 & 0 & 1 & 0 & 0 & 0 & 2 & 1 & 0 & 0 \\
\hline Anemia & 0 & 0 & 0 & 0 & 1 & 0 & 0 & 0 & 1 & 0 & 0 & 0 \\
\hline Fatigue & 0 & 0 & 0 & 0 & 1 & 0 & 0 & 0 & 1 & 0 & 0 & 0 \\
\hline Nausea & 1 & 0 & 0 & 0 & 0 & 0 & 0 & 0 & 1 & 0 & 0 & 0 \\
\hline Anorexia & 1 & 0 & 0 & 0 & 0 & 0 & 0 & 0 & 1 & 0 & 0 & 0 \\
\hline Mucositis & 0 & 0 & 0 & 0 & 2 & 0 & 0 & 0 & 2 & 0 & 0 & 0 \\
\hline Hyperbilirubinemia & 0 & 0 & 0 & 0 & 0 & 1 & 0 & 0 & 0 & 1 & 0 & 0 \\
\hline Epigastric pain & 0 & 0 & 0 & 0 & 1 & 0 & 0 & 0 & 1 & 0 & 0 & 0 \\
\hline Elevated Transaminases & 1 & 1 & 0 & 0 & 1 & 0 & 0 & 0 & 2 & 1 & 0 & 0 \\
\hline Diarrhea & 1 & 0 & 0 & 0 & 2 & 2 & 1 & 0 & 3 & 2 & 1 & 0 \\
\hline
\end{tabular}

$G$ grade of toxicity

${ }^{\mathrm{a}} n=$ number of patients experiencing a given toxicity

\begin{tabular}{|c|c|c|c|c|c|c|c|c|c|c|c|c|}
\hline & \multicolumn{4}{|c|}{ Dose level 1} & \multicolumn{4}{|c|}{ Dose level 2} & \multicolumn{4}{|c|}{ Overall } \\
\hline & \multicolumn{4}{|l|}{$n^{\mathrm{a}}$} & \multicolumn{4}{|l|}{$n^{\mathrm{a}}$} & \multicolumn{4}{|l|}{$n^{\mathrm{a}}$} \\
\hline & G1 & G2 & G3 & G4 & G1 & $\mathrm{G} 2$ & G3 & G4 & G1 & G2 & G3 & G4 \\
\hline Leucopenia & 1 & 1 & 0 & 0 & 1 & 2 & 0 & 0 & 2 & 3 & 0 & 0 \\
\hline Neutropenia & 1 & 2 & 0 & 0 & 2 & 1 & 0 & 0 & 3 & 3 & 0 & 0 \\
\hline Anemia & 0 & 0 & 0 & 0 & 1 & 1 & 0 & 0 & 1 & 1 & 0 & 0 \\
\hline Hypercholesterolemia & 0 & 0 & 0 & 0 & 1 & 0 & 0 & 0 & 1 & 0 & 0 & 0 \\
\hline Hand-foot syndrome & 0 & 1 & 0 & 0 & 1 & 2 & 1 & 0 & 1 & 3 & 1 & 0 \\
\hline Rash & 0 & 1 & 0 & 0 & 1 & 0 & 0 & 0 & 1 & 1 & 0 & 0 \\
\hline Pneumonia & 0 & 0 & 0 & 0 & 1 & 0 & 0 & 0 & 1 & 0 & 0 & 0 \\
\hline Fatigue & 0 & 0 & 0 & 0 & 3 & 2 & 0 & 0 & 3 & 2 & 0 & 0 \\
\hline Nausea & 0 & 0 & 0 & 0 & 1 & 0 & 0 & 0 & 1 & 0 & 0 & 0 \\
\hline Vomiting & 0 & 1 & 0 & 0 & 0 & 0 & 0 & 0 & 0 & 1 & 0 & 0 \\
\hline Anorexia & 0 & 0 & 0 & 0 & 0 & 0 & 1 & 0 & 0 & 0 & 1 & 0 \\
\hline Mucositis & 0 & 2 & 0 & 0 & 3 & 1 & 1 & 0 & 3 & 3 & 1 & 0 \\
\hline Hyperbilirubinemia & 0 & 0 & 0 & 0 & 1 & 1 & 0 & 0 & 1 & 1 & 0 & 0 \\
\hline Epigastric pain & 1 & 0 & 0 & 0 & 1 & 0 & 0 & 0 & 2 & 0 & 0 & 0 \\
\hline Elevated transaminases & 2 & 1 & 0 & 0 & 2 & 1 & 0 & 0 & 4 & 2 & 0 & 0 \\
\hline Diarrhea & 1 & 0 & 0 & 0 & 2 & 2 & 1 & 0 & 3 & 2 & 1 & 0 \\
\hline Hypercreatininemia & 1 & 0 & 0 & 0 & 0 & 0 & 0 & 0 & 1 & 0 & 0 & 0 \\
\hline Hypokalemia & 0 & 0 & 0 & 0 & 1 & 0 & 0 & 0 & 1 & 0 & 0 & 0 \\
\hline Colitis & 0 & 0 & 1 & 0 & 0 & 0 & 0 & 0 & 0 & 0 & 1 & 0 \\
\hline
\end{tabular}

$G$ grade of toxicity

${ }^{\mathrm{a}} n=$ number of patients experiencing a given toxicity (each patient was registered under the maximum grade experienced for each kind of toxicity experienced)
10 cycles, respectively (corresponding to cumulative doses of 390 and $300 \mathrm{mg} / \mathrm{m}^{2}$, respectively), but had no significant changes in LVEF. Two patients had temporary increases in TnT-hs levels above the rule-out value for myocardial damage of $10 \mathrm{ng} / \mathrm{L}$, but well below the rule-in value for myocardial damage of $50 \mathrm{ng} / \mathrm{L}$, with values of 20 and $21 \mathrm{ng} / \mathrm{L}$, respectively, which did not increase over time and were considered not clinically significant (Fig. 1b). All 8 patients evaluable for NT-proBNP had levels within normal limits throughout the study period (Fig. 1c). 

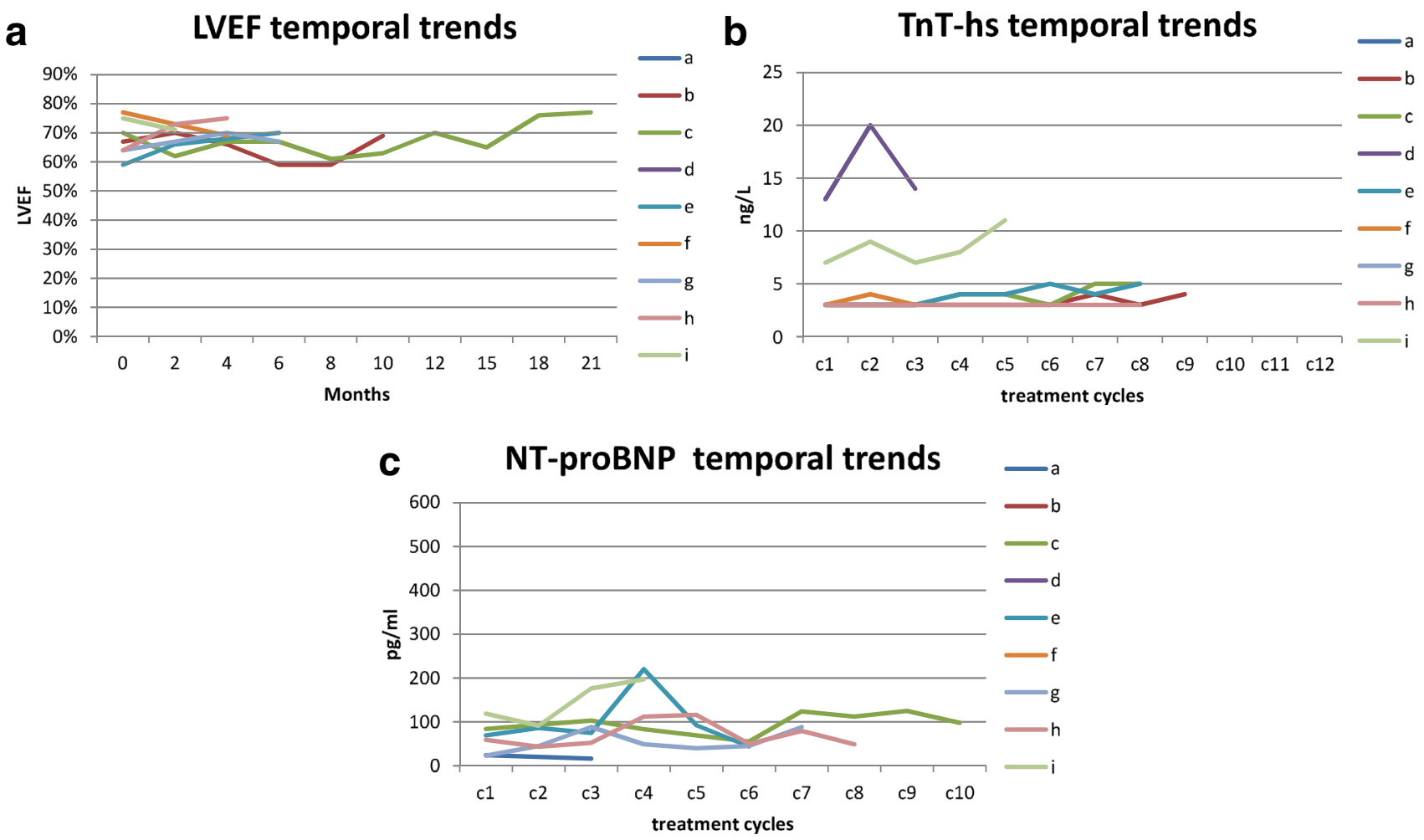

Fig. 1 Temporal trends of cardiac parameters and biomarkers: a left ventricular ejection fraction (LVEF, normal value $\geq 55 \%$ ); $\mathbf{b}$ high-sensitivity troponin $\mathrm{T}$ (TnT-hs, normal value $<10 \mathrm{ng} / \mathrm{L}$; rule-in value for

Temporary interruptions of $\mathrm{L}$ were necessary in eight patients (median 6 days, range 2-24), mainly for diarrhea, mucositis, or skin toxicity; delayed administrations of PLD were necessary in five patients (median 1 week, range 1-4 weeks, with subsequent dose reductions in 2 cases), mainly for diarrhea, mucositis, skin toxicity, or leucopenia.

Definitive interruption of either or both study drugs for reasons other than disease progression occurred in 5 patients: one prudently stopped PLD after a cumulative dose of $390 \mathrm{mg} / \mathrm{m}^{2}$; one stopped PLD after 9 cycles out of a personal choice; one, pretreated with doxorubicin $240 \mathrm{mg} / \mathrm{m}^{2}$, prudently stopped PLD after a cumulative dose of $300 \mathrm{mg} / \mathrm{m}^{2}$, and continued with L plus anastrozole outside the study; one stopped L after the first cycle, because of G3 diarrhea (DLT) recurring also after dose reduction to $1000 \mathrm{mg} /$ day, and stopped PLD for medical decision (later switched to maintenance endocrine treatment) after 7 cycles; one stopped L after 2 cycles due to toxicity (G3 anorexia).

Because of the long-term intent of the treatment, and the occurrence of G3 adverse events beyond the first cycle, we decided to discontinue patient enrollment into dose level 3; dose level 2 was considered as the RP2D.

Median follow-up was 22 months. Best responses were: one partial response, 1 stable disease and 1 disease myocardial damage $>50 \mathrm{ng} / \mathrm{L}$ ); $\mathbf{c}$-terminal pro b-type Natriuretic Peptide (NT-proBNP, normal value $<450 \mathrm{pg} / \mathrm{ml}$ )

progression at dose level 1; four stable diseases and two disease progressions at dose level 2. Overall, six patients had clinical benefit (objective response or disease stabilization $\geq 4$ months). With a median follow-up of 96 weeks, median time to progression was 23 weeks.

\section{Discussion}

Our study shows that combination of PLD and $\mathrm{L}$ is feasible without raising new specific toxicity concerns. In particular, no cardiac adverse events were reported, and an accurate assessment of the circulating cardiac biomarkers TnThs and NT-proBNP, along with ultrasonographic studies of the cardiac function, detected no signs of early cardiac damage. No G4 toxicities occurred; the most important G3 events were expected from the use of the two agents, i.e., hand-foot syndrome and mucositis for PLD and diarrhea for L. Liver toxicity was present but $\leq \mathrm{G} 2$, and no cases of severe drug-induced liver injury were reported. Furthermore, hematological toxicity was never $>\mathrm{G} 2$, ensuring treatment tolerability also in heavily pretreated patients.

The treatment showed hints of activity, with 1 objective response and five disease stabilizations among nine 
evaluable patients, most of whom were pretreated with $\geq 2$ lines of anti-HER 2 therapy for advanced disease.

The pegylated liposomal formulation of doxorubicin favors drug delivery to the tumor, protection from normal organ toxicity, and prolonged half-life [17]. These features contribute to a better therapeutic index than with conventional doxorubicin, as documented by a randomized phaseIII trial showing non inferior progression-free survival (PFS) with PLD, with significantly reduced risk of cardiotoxicity [18]. A better cardiac tolerance was confirmed with endomyocardial biopsies in patients treated with high cumulative doses of PLD [19]. Moreover, PLD was proven to be feasible and active in patients pretreated with neo-/ adjuvant anthracyclines [20, 21].

L, a reversible dual inhibitor of EGFR and HER2 tyrosine kinases, effectively inhibits the main downstream pathways of these receptors, inhibiting cell proliferation and survival, and inducing the expression of pro-apoptotic molecules [22]. In phase-III randomized trials, $\mathrm{L}$ was shown to improve PFS when added to capecitabine or to letrozole in patients with HER2-positive advanced BC [23, 24].

The rate of cardiac events with L, either in monotherapy or in combination with drugs other than anthracyclines, was reported to be $1.6 \%$ in a pooled analysis of 3689 patients [15], with mean time to onset of 13 weeks, partial or full recovery in $88 \%$ of the cases, and rate of symptomatic congestive heart failure of $0.2 \%$. A rate of $2.2 \%$ of cardiac events was observed with $\mathrm{L}$ in patients pretreated with conventional anthracyclines [15]. Data on L are consistent with type II cardiotoxicity, characterized by a usually reversible myocyte dysfunction, without necrosis. On the contrary, anthracyclines lead to myocyte damage and necrosis, mainly as a result of topoisomerase- $2 \beta$ inhibition [25]. The reduced myocyte damage occurring with PLD, compared with conventional anthracyclines, and the limited functional damage associated with L, might allow their combined administration. A combination of trastuzumab with non-pegylated liposomal doxorubicin and paclitaxel has been shown to be tolerable, with a rate of grade 3-4 heart failure of $3 \%$ and treatment discontinuation due to LVEF decrease in $6 \%$ of the patients [26]. The PLD $+\mathrm{L}$ combination could be expected to be less cardiotoxic than the above triplet, although its cardiac safety must be evaluated in adequately powered trials.

Both PLD and L are suitable for long-term treatments, which is particularly crucial in the metastatic setting, and is confirmed by the limited toxicity registered in our study. Nonetheless, safety of long-term administration needs be confirmed in larger studies.

Concerning efficacy and potential synergy or additivity, preclinical studies have shown that L blocks efflux pumps such as the breast cancer resistance protein (BCRP/ ABCG2), leading to increased intracellular accumulation of chemotherapeutic agents when administered concomitantly $[16,27]$. Concomitant treatment of L and PLD showed additive effects in HER2-positive BC cell lines, and a potential synergy in other EGFR-positive, HER2-negative cell lines. Despite some conflicting data [28], this HER2independent mechanism of action could prove useful especially in heterogeneous tumors, where HER2-positive and HER2-negative subpopulations coexist.

The landscape of pharmacological treatment of advanced HER2-positive breast cancer is rapidly evolving, and the potential collocation of a treatment with capecitabine + lapatinib will depend on new drugs becoming available. Anti-HER2 monoclonal antibodies and tyrosine kinase inhibitors differ in their mechanism of action and resistance [29, 30], and can complement each other when used in combination, concomitantly or in sequence. While other tyrosine kinase inhibitors, such as neratinib, are entering the clinical scenario, $\mathrm{L}$ could have a role beyond its combination with capecitabine or letrozole. Because capecitabine can be used in combination with trastuzumab, and could likely be used, in the future, with other drugs like neratinib, a possible use of PLD+lapatinib is in patients pretreated with capecitabine. Patients intolerant to capecitabine, e.g., for dihydropyrimidine dehydrogenase (DPD) polymorphisms increasing its toxicity, could have the opportunity to receive lapatinib in combination with a non fluoropyrimidine agent.

Both L and PLD were proven to cross the blood-brain barrier, an important aspect in HER2-positive tumors which often lead to brain metastases [31, 32].

The small number of patients enrolled is the main limitation of our study. Slow enrollment was due in part to competing studies on newer anti-HER2 drugs, and in part to difficulties in PLD supply, leading to a recommendation from EMA in September 2011 not to start new treatments with the drug (http://www.ema.europa.eu/docs/en_GB/document_library/Medicine_QA/2011/09/WC500111745.pdf), and solved only in April 2013.

The RP2D emerging from our study is PLD $30 \mathrm{mg} / \mathrm{m}^{2}$ every 3 weeks plus L $1500 \mathrm{mg}$ /daily continuously. While PLD has been widely studied (mainly in a first-line setting) at a dose of $50 \mathrm{mg} / \mathrm{m}^{2} / 4$ weeks, results from phase-II [33] and observational [34] studies showed that lower doses could be equally effective and better tolerated, with a dose intensity of $10 \mathrm{mg} / \mathrm{m}^{2} /$ week suggested as the best choice [35]. For this reason, and because we were interested in long-term tolerability of the treatment, we decided not to proceed to dose level 3, and to consider dose level 2 as the RP2D.

A phase-II study with the same combination of PLD $40 \mathrm{mg} / \mathrm{m}^{2} / 4$ weeks plus L $1250 \mathrm{mg}$ daily has been recently reported [36], showing good activity with 54\% overall response rate in 24 patients, and good tolerability. 
Although the intended dose intensity of PLD is the same as in our study ( $10 \mathrm{mg} / \mathrm{m}^{2} /$ week), the dose of $\mathrm{L}$ is slightly lower. Nonetheless, while our study showed that PLD at $10 \mathrm{mg} / \mathrm{m}^{2} /$ week can be combined with $\mathrm{L}$ at $1500 \mathrm{mg} /$ day in the first cycle, the actual median dose intensity of $\mathrm{L}$ during the whole treatment was $<1250 \mathrm{mg} /$ day $(1220 \mathrm{mg} /$ day), even at dose level 2. Therefore, while selected patients may tolerate $\mathrm{L}$ at $1500 \mathrm{mg} /$ day for long periods also in combination with PLD, a dose of $1250 \mathrm{mg} /$ day may be more suitable for most patients. Despite our RP2D for L showed acceptable initial tolerability, dose adjustments may be required to achieve the best therapeutic index.

In conclusion, the limited toxicity of the combination and the hints of activity would support its further development. Given the small and heterogeneous population, larger studies with different design would be needed to draw any conclusion concerning the potential additivity or synergy between the two agents.

Acknowledgements The authors would like to thank Veronica Zanoni and Cristiano Verna for editorial assistance.

Author contributions Andrea Rocca, Dino Amadori, and Oriana Nanni conceived and designed the study; Andrea Rocca, Lorenzo Cecconetto, Alessandro Passardi, Elisabetta Melegari, Roberta Maltoni, Samanta Sarti, Elisabetta Pietri, Alessio Schirone, Anna Fedeli and Marina Faedi provided study materials or patients; Francesco Fabbri was involved in biological characterization; Andrea Rocca, Lorenzo Cecconetto, Alessandro Passardi, Elisabetta Melegari, Roberta Maltoni, Samanta Sarti, Elisabetta Pietri, Alessio Schirone, Anna Fedeli, Marina Faedi, Dino Amadori, Daniele Andreis, Manuela Monti, Oriana Nanni, Francesco Fabbri, Caterina Donati collected data and critically interpreted results; Daniele Andreis and Oriana Nanni performed statistical analyses; Andrea Rocca, Daniele Andreis, Oriana Nanni, and Elisabetta Melegari prepared the manuscript. All authors were involved in reviewing and commenting on the manuscript and approved the final manuscript.

\section{Compliance with ethical standards}

Conflict of interest Andrea Rocca received honoraria for speaker lectures from Novartis Pharmaceuticals Corporation. Lorenzo Cecconetto declares that he has no conflict of interest. Alessandro Passardi declares that he has no conflict of interest. Elisabetta Melegari declares that she has no conflict of interest. Daniele Andreis declares that he has no conflict of interest. Manuela Monti declares that she has no conflict of interest. Roberta Maltoni declares that she has no conflict of interest. Samanta Sarti declares that she has no conflict of interest. Elisabetta Pietri declares that she has no conflict of interest. Alessio Schirone declares that he has no conflict of interest. Francesco Fabbri declares that he has no conflict of interest. Caterina Donati declares that she has no conflict of interest. Oriana Nanni declares that she has no conflict of interest. Anna Fedeli declares that she has no conflict of interest. Marina Faedi declares that she has no conflict of interest. Dino Amadori declares that he has no conflict of interest.

Funding This study was partially funded by GlaxoSmithKline PLC and by Novartis Pharmaceutical Corporation (from March 02 2015), with a research contribution and lapatinib drug provision.
Informed consent Informed consent was obtained from all individual participants included in the study.

Open Access This article is distributed under the terms of the Creative Commons Attribution 4.0 International License (http:// creativecommons.org/licenses/by/4.0/), which permits unrestricted use, distribution, and reproduction in any medium, provided you give appropriate credit to the original author(s) and the source, provide a link to the Creative Commons license, and indicate if changes were made.

\section{References}

1. GLOBOCAN 2012: Ferlay J, Soerjomataram I, Ervik M, Dikshit R, Eser S, Mathers C, Rebelo M, Parkin DM, Forman D, Bray, F. GLOBOCAN 2012 v1.0, cancer incidence and mortality worldwide: IARC CancerBase No. 11 [Internet]. Lyon, France: International Agency for Research on Cancer; 2013. http://globocan. iarc.fr. Accessed on 28 May 2016

2. Prat A, Perou CM (2011) Deconstructing the molecular portraits of breast cancer. Mol Oncol 5:5-23

3. Kennecke H, Yerushalmi R, Woods R, Cheang MC, Voduc D, Speers CH, Nielsen TO, Gelmon K (2010) Metastatic behavior of breast cancer subtypes. J Clin Oncol 28:3271-3277

4. Slamon DJ, Leyland-Jones B, Shak S, Fuchs H, Paton V, Bajamonde A, Fleming T, Eiermann W, Wolter J, Pegram M, Baselga J, Norton L (2001) Use of chemotherapy plus a monoclonal antibody against HER 2 for metastatic breast cancer that overexpresses HER2. N Engl J Med 344:783-792

5. Yeo B, Kotsori K, Mohammed K, Walsh G, Smith IE (2015) Long-term outcome of HER2 positive metastatic breast cancer patients treated with first-line trastuzumab. Breast 24:751-757

6. Balduzzi S, Mantarro S, Guarneri V, Tagliabue L, Pistotti V, Moja L, D'Amico R (2014) Trastuzumab-containing regimens for metastatic breast cancer. Cochrane Database Syst Rev 12(6):CD006242

7. Giordano SH, Temin S, Kirshner JJ, Chandarlapaty S, Crews JR, Davidson NE, Esteva FJ, Gonzalez-Angulo AM, et. al (2014) Systemic therapy for patients with advanced human epidermal growth factor receptor 2-positive breast cancer: American Society of Clinical Oncology clinical practice guideline. J Clin Oncol 32:2078-2099

8. Piccart-Gebhart MJ, Burzykowski T, Buyse M, Sledge G, Carmichael J, Lück HJ et al (2008) Taxanes alone or in combination with anthracyclines as first-line therapy of patients with metastatic breast cancer. J Clin Oncol 26:1980-1986

9. Bartlett JM, McConkey CC, Munro AF, Desmedt C, Dunn JA, Larsimont DP, O'Malley FP et al (2015) Predicting anthracycline benefit: TOP2A and CEP17-not only but also. J Clin Oncol 33:1680-1687

10. Gennari A, Sormani MP, Pronzato P, Puntoni M, Colozza M, Pfeffer U, Bruzzi P (2008) HER2 status and efficacy of adjuvant anthracyclines in early breast cancer: a pooled analysis of randomized trials. J Natl Cancer Inst 100:14-20

11. Swain SM, Whaley FS, Ewer MS (2003) Congestive heart failure in patients treated with doxorubicin: a retrospective analysis of three trials. Cancer 97:2869-2879

12. Mantarro S, Rossi M, Bonifazi M, D’Amico R, Blandizzi C, La Vecchia C, Negri E, Moja L (2016) Risk of severe cardiotoxicity following treatment with trastuzumab: a meta-analysis of randomized and cohort studies of 29,000 women with breast cancer. Intern Emerg Med 11:123-140 
13. Montemurro F, Rossi V, Nolè F, Redana S, Donadio M, Martinello R, Verri E, Valabrega G, Rocca MC, Jacomuzzi ME, Viale G, Sapino A, Aglietta M (2010) Underuse of anthracyclines in women with HER-2+ advanced breast cancer. Oncologist 15:665-672

14. Xing M, Yan F, Yu S, Shen P (2015) Efficacy and cardiotoxicity of liposomal doxorubicin-based chemotherapy in advanced breast cancer: a meta-analysis of ten randomized controlled trials. PLoS One 10:e0133569

15. Perez EA, Koehler M, Byrne J, Preston AJ, Rappold E, Ewer MS (2008) Cardiac safety of lapatinib: pooled analysis of 3689 patients enrolled in clinical trials. Mayo Clin Proc 83:679-586

16. Vannini I, Zoli W, Fabbri F, Ulivi P, Tesei A, Carloni S, Brigliadori G, Amadori D (2009) Role of efflux pump activity in lapatinib/caelyx combination in breast cancer cell lines. Anticancer Drugs 20:918-925

17. Duggan ST, Keating GM (2011) Pegylated liposomal doxorubicin: a review of its use in metastatic breast cancer, ovarian cancer, multiple myeloma and AIDS-related Kaposi's sarcoma. Drugs 71:2531-2558

18. O'Brien ME, Wigler N, Inbar M, Rosso R, Grischke E, Santoro A et al (2004) Reduced cardiotoxicity and comparable efficacy in a phase III trial of pegylated liposomal doxorubicin $\mathrm{HCl}$ (CAELYX/Doxil) versus conventional doxorubicin for first-line treatment of metastatic breast cancer. Ann Oncol 15:440-449

19. Safra T, Muggia F, Jeffers S, Tsao-Wei DD, Groshen S, Lyass O, Henderson R, Berry G, Gabizon A (2000) Pegylated liposomal doxorubicin (doxil): reduced clinical cardiotoxicity in patients reaching or exceeding cumulative doses of $500 \mathrm{mg} / \mathrm{m}^{2}$. Ann Oncol 11:1029-1033

20. Al-Batran SE, Güntner M, Pauligk C, Scholz M, Chen R, Beiss B, Stopatschinskaja S, Lerbs W, Harbeck N, Jäger E (2010) Anthracycline rechallenge using pegylated liposomal doxorubicin in patients with metastatic breast cancer: a pooled analysis using individual data from four prospective trials. Br J Cancer 103:1518-1523

21. Sparano JA, Makhson AN, Semiglazov VF, Tjulandin SA, Balashova OI, Bondarenko et al (2009) Pegylated liposomal doxorubicin plus docetaxel significantly improves time to progression without additive cardiotoxicity compared with docetaxel monotherapy in patients with advanced breast cancer previously treated with neoadjuvant-adjuvant anthracycline therapy: results from a randomized phase III study. J Clin Oncol 27:4522-4529

22. Segovia-Mendoza M, González-González ME, Barrera D, Díaz L, García-Becerra R (2015) Efficacy and mechanism of action of the tyrosine kinase inhibitors gefitinib, lapatinib and neratinib in the treatment of HER2-positive breast cancer: preclinical and clinical evidence. Am J Cancer Res 5:2531-2561

23. Geyer CE, Forster J, Lindquist D, Chan S, Romieu CG, Pienkowski T, Jagiello-Gruszfeld A et al (2006) Lapatinib plus capecitabine for HER2-positive advanced breast cancer. N Eng1 J Med 355:2733-2743

24. Johnston S, Pippen J Jr, Pivot X, Lichinitser M, Sadeghi S, Dieras V et al (2009) Lapatinib combined with letrozole versus letrozole and placebo as first-line therapy for postmenopausal hormone receptor-positive metastatic breast cancer. J Clin Oncol 27:5538-5546

25. Ewer MS, Ewer SM (2011) Cardiotoxicity of anticancer treatments. Nat Rev Cardiol 12:547-558

26. Baselga J, Manikhas A, Cortés J, Llombart A, Roman L, Semiglazov VF, Byakhov M, Lokanatha D, Forenza S, Goldfarb RH, Matera J, Azarnia N, Hudis CA, Rozencweig M (2014) Phase III trial of nonpegylated liposomal doxorubicin in combination with trastuzumab and paclitaxel in HER2-positive metastatic breast cancer. Ann Oncol 25:592-598

27. Perry J, Ghazaly E, Kitromilidou C, McGrowder EH, Joel S, Powles T (2010) A synergistic interaction between lapatinib and chemotherapy agents in a panel of cell lines is due to the inhibition of the efflux pump BCRP. Mol Cancer Ther 9:3322-3329

28. Bhosle J, Kiakos K, Porter AC, Wu J, Makris A, Hartley JA, Hochhauser D (2013) Treatment with gefitinib or lapatinib induces drug resistance through downregulation of topoisomerase II $\alpha$ expression. Mol Cancer Ther 12:2897-2908

29. Moasser MM (2014) Two dimensions in targeting HER2. J Clin Oncol 32:2074-2077

30. Scaltriti M, Rojo F, Ocaña A, Anido J, Guzman M, Cortes J, Di Cosimo S, Matias-Guiu X, Ramon y Cajal S, Arribas J, Baselga $\mathrm{J}$ (2007) Expression of p95HER2, a truncated form of the HER2 receptor, and response to anti-HER 2 therapies in breast cancer. J Natl Cancer Inst 99:628-638

31. Bartsch R, Berghoff A, Pluschnig U, Bago-Horvath Z, Dubsky $P$, Rottenfusser A et al (2012) Impact of anti-HER2 therapy on overall survival in HER2-overexpressing breast cancer patients with brain metastases. Br J Cancer 106:25-31

32. Siegal T, Horowitz A, Gabizon A (1995) Doxorubicin encapsulated in sterically stabilized liposomes for the treatment of a brain tumor model: biodistribution and therapeutic efficacy. $\mathrm{J}$ Neurosurg 83:1029-1037

33. Al-Batran SE, Meerpohl HG, von Minckwitz G, Atmaca A, Kleeberg U, Harbeck N, Lerbs W, Hecker D, Sehouli J, Knuth A, Jager E (2006) Reduced incidence of severe palmar-plantar erythrodysesthesia and mucositis in a prospective multicenter phase II trial with pegylated liposomal doxorubicin at $40 \mathrm{mg} /$ $\mathrm{m}^{2}$ every 4 weeks in previously treated patients with metastatic breast cancer. Oncol 70:141-146

34. Fiegl M, Mlineritsch B, Hubalek M, Bartsch R, Pluschnig U, Steger GG (2011) Single-agent pegylated liposomal doxorubicin (PLD) in the treatment of metastatic breast cancer: results of an Austrian observational trial. BMC Cancer 11:373

35. Verma S, Dent S, Chow BJ, Rayson D, Safra T (2008) Metastatic breast cancer: the role of pegylated liposomal doxorubicin after conventional anthracyclines. Cancer Treat Rev 34:391-406

36. Pircher M, Mlineritsch B, Fridrik MA, Dittrich C, Lang A, Petru E et al (2015) Lapatinib-plus-pegylated liposomal doxorubicin in advanced HER2-positive breast cancer following trastuzumab: a phase II trial. Anticancer Res 35:517-521 